Mathematical Research Letters 6, 43-60 (1999)

\title{
AN ISOMETRIC IMBEDDING THEOREM FOR HOLOMORPHIC BUNDLES
}

\author{
David W. Catlin and John P. D'Angelo
}

\section{Introduction}

The main result in this paper concerns special Hermitian metrics on vector bundles over certain compact complex manifolds. These metrics are not necessarily isometric pullbacks of the Euclidean metric on the universal bundle over a Grassman manifold, but become so after tensoring sufficiently often with a line bundle. The result generalizes to this context a concrete theorem the authors proved in [CD1] about bihomogeneous polynomials on complex Euclidean space $\mathbb{C}^{n}$ that are positive away from the origin. This earlier work is part of a program involving analogues (for complex variables) of Hilbert's seventeenth problem; the current work both generalizes the earlier work and gives an algebraic geometric context for it.

In order to state our result we use the following notation: $\mathbb{G}_{p, N}$ denotes the Grassman manifold of $p$-dimensional planes in $\mathbb{C}^{N}, \pi: \mathbb{U}_{p, N} \rightarrow \mathbb{G}_{p, N}$ denotes the universal bundle over $\mathbb{G}_{p, N}$, and $g_{0}$ denotes the Euclidean metric on it. Definition 1 gives the precise meaning for an Hermitian metric $R$ on a holomorphic vector bundle $E$ over a complex manifold $M$ to be globalizable. One of the conditions is that $R$ extends to be a complex-valued function on $E \times E$; this enables us to evaluate $R(u, v)$ even when $u$ and $v$ have different base points. We also assume that the extended function is holomorphic in its first variable and Hermitian symmetric. Given holomorphic bundles over $M$ with globalizable Hermitian metrics $G_{1}$ and $G_{2}$, the tensor product bundle has a natural globalizable Hermitian metric written $G_{1} G_{2}$. See Remark 1. The sharp globalizable Cauchy-Schwarz inequality (SGCS) appears in Definition 2. Both (SGCS) and the negativity of $L$ are necessary hypotheses in Theorem 1 . We discuss this in Section 2.

We prove the following result about isometric imbedding:

Received August 27, 1998.

Catlin supported by NSF grant DMS 94015480. D'Angelo supported by NSF grant DMS 9304580 at IAS and by MSRI. 
Theorem 1. Suppose that $M$ is a compact complex manifold. Let $E$ be a vector bundle of rank $p$ over $M$ with globalizable Hermitian metric $G$. Let $L$ be a line bundle over $M$ with globalizable Hermitian metric $R$, and suppose that $L$ is negative and that $R$ satisfies (SGCS). Then there is an integer $d_{0}$ so that, for all $d$ with $d \geq d_{0}$, there is a holomorphic imbedding $h_{d}$ with $h_{d}: M \rightarrow \mathbb{G}_{p, N}$ so that $E \otimes L^{d}=h_{d}^{*} \mathbb{U}_{p, N}$ and $G R^{d}=h_{d}^{*}\left(g_{0}\right)$.

The new part of the conclusion of Theorem 1 is that $G R^{d}=h_{d}^{*}\left(g_{0}\right)$. The classical Kodaira theorem yields an imbedding; we show (given our necessary hypotheses) that this imbedding can be chosen to be an isometry. To help motivate this theorem, we reinterpret it when $M$ is complex projective space, $E$ is a power $\mathbb{U}^{m}$ of the universal line bundle, $L=\mathbb{U}$, and the metric on $\mathbb{U}^{m}$ arises from a bihomogeneous polynomial $p(z, \bar{z})$. The conclusion amounts to saying that there is an integer $d_{0}$ so that, for each $d \geq d_{0}$, there is a holomorphic homogeneous polynomial mapping $h_{d}$ such that

$$
\|z\|^{2 d} p(z, \bar{z})=\left\|h_{d}(z)\right\|^{2} \text {. }
$$

This is one of principal results in [CD1]. Thus we can write a bihomogeneous polynomial on $\mathbb{C}^{n}$, that is strictly positive away from the origin, as a quotient of squared norms of holomorphic polynomial mappings. We remark here that the minimum such integer $d$ can be arbitrarily large even for bihomogeneous polynomials $p$ of degree four.

Non-negative bihomogeneous polynomials cannot necessarily be written as the quotient of such squared norms. In Section 7 we give two examples and briefly discuss two necessary conditions. We hope to determine in future work precisely which non-negative polynomials can be written as quotients of squared norms of holomorphic polynomials. Such a result would give a complex variables analogue of Hilbert's seventeenth problem. The famous solution to this problem by E. Artin (See [R] for a survey) states that a non-negative homogeneous polynomial on real Euclidean space is necessarily a sum of squares of rational functions. Applications from [CD1] suggest why we consider instead whether non-negative polynomials are quotients of squared norms of holomorphic polynomials mappings.

The main result in this paper generalizes Theorem 1 from [CD1]. After the submission of this paper, it was pointed out to the second author that Quillen [Q] had already proved that result. The other results in [CD1] and those in this paper develop new directions and we hope that they are new. The second author acknowledges Dan Grayson for this information.

The main theorem in this paper considers Hermitian metrics on vector bundles, so its concrete analogues imply results about the holomorphic factorization of matrices of real analytic functions. In particular this gives a weak version of Hilbert's seventeenth problem for matrix-valued polynomials. 
Corollary. Suppose that $M(z, \bar{z})$ is a matrix of bihomogeneous polynomials that is positive-definite away from the origin. Suppose $R$ is a bihomogeneous polynomial that is positive away from the origin and satisfies (SGCS). Assume also that $\{R<1\}$ is a strongly pseudoconvex domain. Then there is an integer $d$ and a matrix $A$ of holomorphic homogeneous polynomials such that

$$
R(z, \bar{z})^{d} M(z, \bar{z})=A(z)^{*} A(z) .
$$

In particular we can choose $R(z, \bar{z})=\|z\|^{2 d}$.

Although we cannot in general write $M(z, \bar{z})=A(z)^{*} A(z)$, where $A$ depends holomorphically on $z$, the Corollary enables us to write $R(z, \bar{z})^{d} M(z, \bar{z})$ in this form when $d$ is sufficiently large. Here the symbol $*$ denotes the conjugate transpose. The main theorem in this paper generalizes this by replacing the monomials $z^{\alpha}$ (sections of a power of the hyperplane bundle) by sections $\phi_{\alpha}$ of other bundles.

We conclude the introduction by briefly discussing the proof of Theorem 1 . We first show that the desired pullback property for the natural metric on $E \otimes L^{d}$ follows from a certain matrix of constants being positive-definite. This is equivalent to an integral operator being positive. We use the unit disk bundle to consider these operators for all $d$ simultaneously. We then use properties of the Bergman projection for sections of a vector bundle and facts about compact operators on Hilbert spaces in order to prove positivity modulo compact operators. This shows, when $d$ is sufficiently large, that the metric on $E \otimes L^{d}$ is a pullback of the Euclidean metric on some $\mathbb{U}_{p, N}$.

In Section 2 we discuss why each hypothesis in Theorem 1 is necessary for the conclusion.

The first author acknowledges Laszlo Lempert and the second author acknowledges Steve Bradlow and Yum-Tong Siu for useful discussions.

\section{Globalizable Metrics}

We begin by studying the Euclidean metric on the universal bundle over the Grassman manifold. Let $\mathbb{G}_{p, N}$ denote the Grassman manifold of $p$ planes in complex $N$-space. When $p=1$ we have complex projective space, and we write as usual $\mathbb{P}^{N-1}$ for $\mathbb{G}_{1, N}$. Let $\mathbb{U}_{p, N}$ denote the universal bundle over $\mathbb{G}_{p, N}$. This bundle is sometimes known as the tautological bundle; a point in $\mathbb{U}_{p, N}$ is a pair $(S, \zeta)$ where $S$ is a $p$-dimensional subspace of $\mathbb{C}^{N}$ and $\zeta \in S$. We let $g_{0}$ denote the Euclidean metric on $\mathbb{U}_{p, N}$. By definition we have

$$
g_{0}((S, u),(S, v))=v^{*} u=\langle u, v\rangle=\sum u_{j} \bar{v}_{j} .
$$

In (1), the Euclidean inner product $\langle u, v\rangle$ makes sense because we consider $u, v$ as elements of $\mathbb{C}^{N}$. Alternatively, in terms of a local frame $e$ of $\mathbb{U}_{p, N}$, we define 
$g_{0}(e)=e^{*} e$. We have $\left(g_{0}\right)_{i j}=\left\langle e_{j}, e_{i}\right\rangle$ where $\langle$,$\rangle denotes the usual Hermitian$ inner product on complex Euclidean space $\mathbb{C}^{N}$, and the vectors $e_{i}$ for $i=1, \ldots, r$ are linearly independent. Note the interchange of indices. From this point of view we see that the matrix of $g_{0}$ is of the form $A^{*} A$.

We next make a simple but crucial observation. Consider the function $g_{0}^{\prime}$ : $\mathbb{U}_{p, N} \times \mathbb{U}_{p, N} \rightarrow \mathbb{C}$ defined by

$$
g_{0}^{\prime}\left(\left(S_{1}, v_{1}\right),\left(S_{2}, v_{2}\right)\right)=\left\langle v_{1}, v_{2}\right\rangle .
$$

Then $g_{0}$ is holomorphic in the first variable, anti-holomorphic in the second, satisfies $g_{0}^{\prime}(\alpha, \beta)=\overline{g_{0}^{\prime}(\beta, \alpha)}$, and extends the metric in the sense that $g_{0}^{\prime}(\alpha, \beta)=$ $g_{0}(\alpha, \beta)$ when $\pi(\alpha)=\pi(\beta)$. Henceforth we drop the prime from the notation, and write $g_{0}$ for this function. The crucial fact about the Euclidean metric on the universal bundle is that it makes sense to write $g_{0}(\alpha, \beta)$ even when $\alpha$ and $\beta$ are based at different points.

Suppose that $h: M \rightarrow \mathbb{G}_{p, N}$ is a holomorphic mapping. The pullback $\pi^{\prime}$ : $h^{*}\left(\mathbb{U}_{p, N}\right) \rightarrow M$ is then a bundle over $M$; a point in $h^{*}\left(\mathbb{U}_{p, N}\right)$ is a pair $(z, u)$, where $z \in M, u \in \mathbb{U}_{p, N}$, and $h(z)=\pi(u)$. Write $\alpha$ and $\beta$ for points in $h^{*}\left(\mathbb{U}_{p, N}\right)$. Writing $h_{*}$ as usual for the map satisfying $h \pi^{\prime}=\pi h_{*}$, there is a natural metric $h^{*}\left(g_{0}\right)$ defined on $h^{*}\left(\mathbb{U}_{p, N}\right)$ by

$$
h^{*}\left(g_{0}\right)(\alpha, \beta)=g_{0}\left(h_{*} \alpha, h_{*} \beta\right) .
$$

We see again that $h^{*}\left(g_{0}\right)$ makes sense when $\alpha$ and $\beta$ are based at different points; we can therefore extend the definition of the metric to a function $h^{*}\left(g_{0}\right)$ : $h^{*}\left(\mathbb{U}_{p, N}\right) \times h^{*}\left(\mathbb{U}_{p, N}\right)$ as above. The metrics $g_{0}$ and $h^{*}\left(g_{0}\right)$ are examples of what we call globalizable metrics. For a vector bundle $E$ over $M$ we write $E^{*}$ for its dual bundle and we write $H\left(M, E^{*}\right)$ for the holomorphic sections of $E^{*}$. When $M$ is compact, $H\left(M, E^{*}\right)$ is a finite-dimensional complex vector space.

Definition 1. Let $\pi^{\prime}: E \rightarrow M$ be a holomorphic vector bundle over a complex manifold $M$. We suppose that $G$ is an Hermitian metric on $E$. We say that $G$ is globalizable if there is a mapping $G^{\prime}: E \times E \rightarrow \mathbb{C}$ such that the following properties hold:

1. $G^{\prime}$ extends the metric: $G^{\prime}(u, v)=G(u, v)$ whenever $\pi^{\prime}(u)=\pi^{\prime}(v)$.

2. $G^{\prime}$ is holomorphic in the first variable: $G^{\prime}(\cdot, v) \in H\left(M, E^{*}\right)$.

3. $G^{\prime}$ is Hermitian: $G^{\prime}(u, v)=\overline{G^{\prime}(v, u)}$.

Henceforth we will write $G$ instead of $G^{\prime}$. For a fixed $u$, the map $v \rightarrow G(u, v)$ is anti-holomorphic. Suppose that $\phi_{1}, \ldots, \phi_{q}$ denote a basis of $H\left(M, E^{*}\right)$. We can write

$$
G(u, v)=\sum_{j}\left\langle G(u, v), \phi_{j}\right\rangle \phi_{j}
$$


and hence (for this fixed $u)\left\langle G(u, v), \phi_{j}\right\rangle$ defines an element of $\overline{H\left(M, E^{*}\right)}$. This gives

$$
G(u, v)=\sum_{j, k=1}^{q} G_{k j} \phi_{j}(u) \overline{\phi_{k}(v)}
$$

for constants $G_{k j}$. (The domain of the function $\phi_{j}$ is $M$. When $\pi^{\prime}(u)=z$ we write $\phi_{j}(u)$ for the value of the linear functional $\phi_{j}(z)$ on $u_{z}$.)

For clarity we repeat the conclusion. Suppose $G$ is globalizable and $\phi_{1}, \ldots, \phi_{q}$ form a basis for $H\left(M, E^{*}\right)$. Then there is a matrix $G_{k j}$ of constants so that (4) holds.

We note immediately that not all bundles $E$ admit globalizable metrics. A necessary and sufficient condition is that, for each non-zero vector $v \in E$ there is a section $\phi$ of $E^{*}$ with $\phi(v) \neq 0$. The collection of these sections determines a holomorphic map to some $\mathbb{G}_{p, N}$.

Remark 1. Suppose for $j=1,2$ that $G_{j}$ is a globalizable Hermitian metric on a holomorphic vector bundle $E_{j}$ over a complex manifold $M$. Then the formula

$$
G\left(u_{1} \otimes u_{2}, v_{1} \otimes v_{2}\right)=G_{1}\left(u_{1}, v_{1}\right) G_{2}\left(u_{2}, v_{2}\right)
$$

determines a globalizable metric $G$ on $E_{1} \otimes E_{2}$. It is natural to write $G=G_{1} G_{2}$.

Suppose that $G$ is globalizable, and that (4) holds. By property 3 of Definition 1 , the matrix of coefficients $\left(G_{k j}\right)$ must be Hermitian. It can have eigenvalues of both signs. Consider the case when it is positive-definite. In this case we can think of $G$ as defining an Hermitian inner product on $H\left(M, E^{*}\right)$ and we obtain a holomorphic mapping from $M$ to $\mathbb{G}_{p, q}$ inducing this metric. To see this concretely, suppose that $\left(G_{k j}\right)$ is a positive-definite matrix. Then there are $q$ independent vectors $\zeta_{k}$ in $\mathbb{C}^{q}$ so that $G_{k j}=\left\langle\zeta_{j}, \zeta_{k}\right\rangle$, and hence, from (4),

$$
G(u, v)=\sum_{j, k=1}^{q}\left\langle\zeta_{j}, \zeta_{k}\right\rangle \phi_{j}(u) \overline{\phi_{k}(v)}=\left\langle\sum \zeta_{j} \phi_{j}(u), \sum \zeta_{k} \phi_{k}(v)\right\rangle
$$

We can use (6) to construct a holomorphic map $h: M \rightarrow \mathbb{G}_{p, q}$. If we write $\psi(u)=\sum \zeta_{j} \phi_{j}(u)$, then $\psi$ defines a map from $E$ to $\mathbb{C}^{q}$. For $z \in M$, write $E_{z}$ for the fibre over $z$. Then, since $\psi$ is injective on each fibre, $\psi\left(E_{z}\right)$ is a $p$-dimensional subspace of $\mathbb{C}^{q}$; we write $h(z)=\psi\left(E_{z}\right)$. We see from (6) that $G(u, v)=g_{0}\left(h_{*}(u), h_{*}(v)\right)$. Thus, as $u_{z}$ varies over the $p$-dimensional fibre $E_{z}$, the formula $h(z)=\sum \phi_{j}(z)\left(u_{z}\right) \zeta_{j}$ defines a $p$-dimensional subspace of $\mathbb{C}^{q}$.

In Section 2 we will discuss circumstances under which the mapping $h$ is an imbedding. We summarize our discussion so far in the next proposition. 
Proposition 1. Let $G$ be a globalizable Hermitian metric on a holomorphic vector bundle $V$ over $M$. Suppose that the matrix $G_{k j}$ defined by (4) is positivedefinite. Then $G=h^{*}\left(g_{0}\right)$ for some holomorphic mapping $h: M \rightarrow \mathbb{G}_{p, N}$. Conversely, the pullback of a holomorphic map $h: M \rightarrow \mathbb{G}_{p, N}$ defines a globalizable metric on $h^{*}\left(\mathbb{U}_{p, N}\right)$ by formula $(3)$.

We discuss Proposition 1 in the special case when $M=\mathbb{P}^{n-1}$ and $V=\mathbb{U}_{1, N}^{m}=$ $\mathbb{U}^{m}$. The sections of the dual bundle are then the homogeneous monomials $z^{\alpha}$, and the globalizable metric $G$ can be written

$$
G(z, w)=\sum_{|\alpha|=|\beta|=m} c_{\beta \alpha} z^{\alpha} \bar{w}^{\beta}
$$

Let $p$ be a polynomial defined on $\mathbb{C}^{n}$; we write its values as $p(z, \bar{z})$. When $p$ is positive away from the origin and bihomogeneous of degree $2 m$, it determines a globalizable metric on $\mathbb{U}^{m}$. We check that $p$ defines a metric using the transition functions of the bundle. In the open coordinate chart of $\mathbb{P}^{n-1}$ where $z_{j} \neq 0$, we define $G_{j}$ by

$$
G_{j}(z, \bar{z})=\frac{p(z, \bar{z})}{\left|z_{j}\right|^{2 m}}
$$

Each $G_{j}$ is positive where it is defined and $G_{k}=\left|\left(\frac{z_{j}}{z_{k}}\right)^{m}\right|^{2} G_{j}$. Since the functions $\left(\frac{z_{j}}{z_{k}}\right)^{m}$ are the transition functions for $\mathbb{U}^{m}$, the collection of functions $G_{k}$ define a metric on $\mathbb{U}^{m}$. The three conditions needed for it to be globalizable follow immediately.

We next observe that it is possible for $G(z, \bar{z})$ to be positive for all $z \in \mathbb{C}^{n}$ with $z \neq 0$, but yet the underlying matrix $\left(c_{\beta \alpha}\right)$ need not be. This shows that such a metric is not necessarily a pullback of the Euclidean metric, and indicates the content of the theorem.

Example. Put $p(z, \bar{z})=\left|z_{1}\right|^{4}+c\left|z_{1} z_{2}\right|^{2}+\left|z_{2}\right|^{4}$ with $c>-2$. Then the matrix of coefficients (with respect to the basis $z_{1}^{2}, z_{1} z_{2}, z_{2}^{2}$ ) is diagonal, with eigenvalues $1, c, 1$. Yet $p$ is obviously positive away from the origin. In this example it is easy to prove that the minimum $d$ required (in Theorem 1) for a given $c$ tends to infinity as $c$ tends to -2 .

\section{Why the hypotheses of Theorem 1 are necessary}

In this section we show that pullbacks of the Euclidean metric on the universal bundle over Grassmanians satisfy certain properties, and therefore that these properties must be hypothesized in Theorem 1. We also show how these conditions are related to $h_{d}$ being an imbedding. 
Definition 2. Let $L$ be a line bundle over $M$ with globalizable Hermitian metric $R$. Then $R$ satisfies the global Cauchy-Schwarz inequality (GCS) if

$$
\left|R\left(u_{1}, u_{2}\right)\right|^{2} \leq R\left(u_{1}, u_{1}\right) R\left(u_{2}, u_{2}\right)
$$

and $R$ satisfies the sharp global Cauchy-Schwarz inequality (SGCS) if

$$
\left|R\left(u_{1}, u_{2}\right)\right|^{2}<R\left(u_{1}, u_{1}\right) R\left(u_{2}, u_{2}\right)
$$

holds whenever $u_{1} \neq u_{2}$ and their vector parts are non-zero.

Definition 3. We say that $L$ is negative if the unit disk $B=\{z: R(z, z)<1\}$ is a strongly pseudoconvex domain in $L$.

See $[\mathrm{D}, \mathrm{W}]$ for various other definitions of negative. We next discuss why these properties are required as necessary conditions in Theorem 1 for the line bundle $L$.

Let $g$ be a fibre metric on a Hermitian vector bundle $E$ over a complex manifold $M$. We write $\operatorname{Ric}(g)$ for the Ricci curvature form $\bar{\partial} \partial\left(\log (\operatorname{Det}(g))\right.$. Let $g_{0}$ be the Euclidean metric on the universal bundle; its Ricci curvature form is negative. Thus for nonzero tangent vectors $v$ we have $\operatorname{Ric}\left(g_{0}\right)(v, v)<0$.

Proposition 2. Suppose that $M$ is a compact complex manifold, and that $h$ : $M \rightarrow \mathbb{G}_{p, N}$ is a holomorphic mapping. Let $E=h^{*}\left(\mathbb{U}_{p, N}\right)$ denote the pullback bundle, and assume we are given the pullback metric $h^{*}\left(g_{0}\right)$ on $E$. Then

1. $h$ is an immersion if and only if the $(1,1)$ form $\operatorname{Ric}\left(h^{*}\left(g_{0}\right)\right)$ is negative.

2. $h$ is injective if and only if for all distinct points $z, w$ in $M$, there is $u \in E_{z}$ such that, for all nonzero $v \in E_{w}$, the (SGCS) inequality

$$
\left|h^{*}\left(g_{0}\right)(u, v)\right|^{2}<\left|h^{*}\left(g_{0}\right)(u, u)\right|^{2}\left|h^{*}\left(g_{0}\right)(v, v)\right|^{2}
$$

holds.

Proof. By functorial properties of the curvature, $\operatorname{Ric}\left(h^{*}\left(g_{0}\right)\right)=h^{*} \operatorname{Ric}\left(g_{0}\right)$. Thus we have, for a holomorphic tangent vector $L$ of $M$,

$$
\operatorname{Ric}\left(h^{*}\left(g_{0}\right)\right)(L, L)=\operatorname{Ric}\left(g_{0}\right)(\partial h(L), \partial h(L)) .
$$

If $h$ is an immersion, and $L \neq 0$, then $\partial h(L) \neq 0$, and the right side is negative. Thus the left side is negative. Conversely if the left side is negative for all nonzero $L$, then $\partial h(L) \neq 0$ for all nonzero $L$, and $h$ is an immersion. This proves the first statement.

To prove the second statement, suppose first that $h$ is injective. Choose distinct points $z$ and $w$; hence $h(z) \neq h(w)$. Choose $u \in E_{z}$ so that $h_{*}(u)$ is not in $h_{*}\left(E_{w}\right)$. If $v \in E_{w}$ is nonzero, then $h_{*}(u)$ is not a multiple of $h_{*}(v)$. Hence

$$
\left|h^{*}\left(g_{0}\right)(u, v)\right|^{2}=\left|g_{0}\left(h_{*}(u), h_{*}(v)\right)\right|^{2}<g_{0}\left(h_{*}(u), h_{*}(u)\right) g_{0}\left(h_{*}(v), h_{*}(v)\right)
$$


by the Cauchy-Schwarz inequality for the Euclidean metric $g_{0}$. Thus (9) holds.

Conversely, suppose that there is such a $u$. Then $h_{*}(u)$ is not a multiple of $h_{*}(v)$ for any $v \in E_{w}$. Hence $h_{*}\left(E_{z}\right) \neq h_{*}\left(E_{w}\right)$ and hence $h(z) \neq h(w)$.

We use this result to show why the conditions on the globalizable metric $R$ in Theorem 1 are necessary. If the conclusion of Theorem 1 holds for all vector bundles $E$ satisfying the hypotheses, then it holds in particular when $E=L$.

Suppose that $L$ is a line bundle over $M$ with globalizable Hermitian metric $R$. There is a natural metric $R^{d}$ defined on $L^{d}$ by using Remark 1 . We amplify this point here. Since the fibres are one-dimensional it suffices to give the definition of $R^{d}(u, v)$ when the vector parts of $u$ and $v$ are $d$-fold tensor products. Suppose that $\left(z_{1}, l_{1}^{d}\right)=u$ and $\left(z_{2}, l_{2}^{d}\right)=v$ are local representations of points in $L^{d}$. We put

$$
R^{d}(u, v)=R^{d}\left(\left(z_{1}, l_{1} \otimes \ldots \otimes l_{1}\right),\left(z_{2}, l_{2} \otimes \ldots \otimes l_{2}\right)\right)=R\left(\left(z_{1}, l_{1}\right),\left(z_{2}, l_{2}\right)\right)^{d} .
$$

Suppose that there is a holomorphic imbedding $h_{d}: M \rightarrow \mathbb{P}^{N}$, and that $R^{d}=h_{d}^{*}\left(g_{0}\right)$. Here $N+1$ is the dimension of the space of holomorphic sections of $\left(L^{*}\right)^{d}$. Then we claim that (SGCS) must hold for $R$, and the curvature of $R$ must be negative.

First we show that (SGCS) holds. We write $l^{d}$ for the $d$-fold tensor product. Since $h_{d}$ is assumed to be injective, Proposition 2 implies that

$$
\left|R^{d}\left(l_{1}^{d}, l_{2}^{d}\right)\right|^{2}<R^{d}\left(l_{1}^{d}, l_{1}^{d}\right) R^{d}\left(l_{2}^{d}, l_{2}^{d}\right),
$$

and hence that

$$
\left|R\left(l_{1}, l_{2}\right)\right|^{2 d}<R\left(l_{1}, l_{1}\right)^{d} R\left(l_{2}, l_{2}\right)^{d} .
$$

Taking $d$-th roots of (12) yields (SGCS).

Next we show that $R$ has negative curvature. Since $h_{d}$ is an immersion, Proposition 2 implies that $\operatorname{Ric}\left(R^{d}\right)$ is negative. $\operatorname{Since} \operatorname{Ric}\left(R^{d}\right)=d \operatorname{Ric}(R)$ we see that $\operatorname{Ric}(R)$ is also negative.

Remark 2. It is well known that the negativity of the curvature is equivalent to the unit ball $B$ in $L$ being strongly pseudoconvex. When $R^{d}=h^{*}\left(g_{0}\right)$, we see that $B$ is defined by the equation

$$
r(u)=g_{0}\left(h_{*} u, h_{*} u\right)=\|H(u)\|^{2}<1 .
$$

The domain $B$ will be strongly pseudoconvex if the complex Hessian of a defining function is positive-definite on the boundary. Choosing $a \in \mathbb{C}^{n}$, we have

$$
\partial \bar{\partial} r(a, a)=\|\partial H(a)\|^{2} \geq c\|a\|^{2}
$$


whenever $\partial H$ has maximal rank. Equation (14) shows that $B$ is strongly pseudoconvex whenever $h$ is an immersion.

We collect the information we have proved about (SGCS) and the negativity of $L$ in the following proposition.

Proposition 3. Let $M$ be a complex manifold and let $L$ be a line bundle over $M$ with Hermitian metric $R$. Suppose that there is an integer $d$ and a holomorphic immersion $h_{d}$ to $\mathbb{P}^{N}$ such that $L^{d}=h^{*}\left(\mathbb{U}_{1, N}\right)$ and such that $R^{d}=h^{*}\left(g_{0}\right)$. Then $L$ is negative and (GCS) holds. If also $h_{d}$ is injective, then (SGCS) holds.

Proposition 3 has a concrete corollary; this is the special case again when $L$ is a power of the universal bundle and the metric is given by a bihomogeneous polynomial.

Corollary 1. Suppose that $r(z, \bar{z})$ is a bihomogeneous polynomial of degree $2 k$ that is positive away from the origin. Suppose that, for every positive bihomogeneous polynomial $f$, there is an integer $d$ such that $f r^{d}$ is a squared norm of a holomorphic mapping. Then the domain $\Omega=\{r(z, \bar{z})<1\}$ must be strongly pseudoconvex. Furthermore, the (GCS) inequality $|r(z, \bar{w})|^{2} \leq r(z, \bar{z}) r(w, \bar{w})$ must hold.

One can prove Corollary 1 without reference to bundles. The (GCS) inequality is proved as above. We prove negativity by contradiction. If the domain $\{r<1\}$ is not strongly pseudoconvex, then it has a weakly pseudoconvex boundary point $p$. One can then find a non-zero tangent vector $L$ at $p$ of type $(1,0)$ so that $\bar{L} \operatorname{Lr}(p)=0$. Let $z^{\beta}$ be a monomial with $L\left(z^{\beta}\right)(p) \neq 0$. For positive $\epsilon$ define $f$ by $f(z, \bar{z})=r(z, \bar{z})-\epsilon\left|z^{\beta}\right|^{2}$. When $\epsilon$ is sufficiently small, $f$ is positive on the sphere. A simple computation shows that $f r^{d}$ cannot be a squared norm. To see this, we compute $(\bar{L} L) f r^{d}(p)$. Since $L(r)(p)=\bar{L}(r)(p)=0$, the only non-zero term arises when both derivatives hit $f$, and this term is negative. Thus the Hessian of $f r^{d}$ has a negative eigenvalue at $p$, and hence $f r^{d}$ is not a squared norm. Since $d$ is arbitrary, this proves the Corollary directly.

\section{The Bergman kernel}

We return to the general situation. The data in Theorem 1 are a compact complex manifold $M$, a line bundle $L$ over $M$, and a vector bundle $E$ over $M$. We are given a globalizable metric $R$ on $L$, and a globalizable metric $G$ on $E$. As above we consider the natural metric written $R^{d} G$ on $E \otimes L^{d}$. In order to prove Theorem 1, we will apply Proposition 1 . We need to find an integer $d$ such that the metric $R^{d} G$ can be expressed by

$$
R^{d} G=\sum G_{k j} \phi_{j} \overline{\phi_{k}}
$$


where the matrix $G_{k j}$ is positive-definite and the $\phi_{j}$ form a basis for $H(M,(E \otimes$ $\left.\left.L^{d}\right)^{*}\right)$. In order to do this, we will consider all the spaces $H\left(M,\left(E \otimes L^{d}\right)^{*}\right)$ at the same time.

We let $B$ be the unit disk bundle defined by $B=\{z \in L: R(z, z)<1\}$. Let $p: B \rightarrow M$ denote the projection map, and let $\mathcal{E}=p^{*}(E)$; we think of $\mathcal{E}$ as a bundle over $B$. We obtain a globalizable pullback metric $\mathcal{G}=p^{*}(G)$. By definition

$$
\mathcal{G}(\eta, \tau)=p^{*}(G)(\eta, \tau)=G\left(p_{*} \eta, p_{*} \tau\right) .
$$

We let $\langle$,$\rangle denote an Hermitian metric on \mathcal{E}^{*}$ that is invariant under the automorphism of $B$ corresponding to multiplication by $e^{i \theta}$. The metric $R$ determines a volume form $d V$ on $B$.

Let $g$ be a holomorphic section of $\mathcal{E}^{*}$ over $B$. A point in $B$ is a pair $(z, u)$ where $z \in M$ and $u$ is one-dimensional. We say that $g \in \mathcal{H}^{d}\left(B, \mathcal{E}^{*}\right)$ if $g$ is homogeneous of degree $d$ with respect to the second variable $u$. These spaces are then orthogonal because the metric on $\mathcal{E}^{*}$ is invariant. The disk bundle construction enables us to expand $g$ in a manner analogous to writing a convergent power series as a sum of homogeneous polynomials. We can identify $\mathcal{H}^{d}\left(B, \mathcal{E}^{*}\right)$ with holomorphic sections of $\left.\left(E \otimes L^{d}\right)\right)^{*}$ and thus write $g=\sum_{0}^{\infty} g_{j}$ as its homogeneous expansion. ( $\mathcal{H}$ stands for "homogeneous" and $\mathcal{H}^{d}$ does not mean cohomology.)

We need some results about the Bergman projection for sections of a vector bundle. The first author proved these results in $[\mathrm{C}]$.

Suppose that $\Omega$ is a strongly pseudoconvex domain in a complex manifold. We suppose that $\Omega$ has smooth boundary, and we write $\bar{\Omega}$ for the closure of $\Omega$. Let $\pi: V \rightarrow \bar{\Omega}$ be a complex vector bundle of rank $p$. Assume that $g$ is a Hermitian metric on $T \Omega$ and that $G$ is a Hermitian metric on $V$. Let $P: L^{2}(\Omega, V) \rightarrow A^{2}(\Omega, V)$ denote the Bergman projection. The Bergman kernel function $K$ is defined so that $K(z, w)$ is the element of $\operatorname{Hom}\left(V_{w}, V_{z}\right)$ for which

$$
(P f)(z)=\int_{\Omega} K(z, w) f(w) d V(w) .
$$

We want to find an approximate formula for $K(z, w)$ in terms of a defining function $R$ for $\Omega$. In our situation $\Omega$ has real-analytic boundary, and $\Omega$ is defined by the inequality $R(z, z)<1$. Let $c_{n}$ denote the constant $\frac{n !}{2^{n+1} \pi^{n}}$. We write $\operatorname{det}(\partial \bar{\partial} R)_{z}$ for the determinant of the complex Hessian of $R$; it is computed in terms of a basis of the holomorphic tangent space to $b \Omega$ at $z$ that is orthonormal with respect to the metric $g$. We also compute $|d R(z)|_{g}$ with respect to $g$. For convenience we write

$$
\lambda(z)=c_{n}(\operatorname{det} \partial \bar{\partial} R)_{z}|d R(z)|_{g}^{2} .
$$

We can now write down the approximate form of the Bergman kernel function. This result is a vector bundle analogue of one part of the description of the 
Bergman kernel function for strongly pseudoconvex domains from $[\mathrm{F}]$ and $[\mathrm{BS}]$. See $[\mathrm{C}]$ for a proof.

Proposition 4. Assume that $\Omega$ is a strongly pseudoconvex domain in a complex manifold of dimension $n$ as above. Let $P: L^{2}(\Omega, V) \rightarrow A^{2}(\Omega, V)$ denote the Bergman projection. Suppose that $s=s_{1}, \ldots, s_{p}$ is a local frame for $V$ over an open set $W$ in $\bar{\Omega}$, and let $W^{\prime} \subset \subset W$ be a relatively compact subset. Write $f \in L^{2}\left(W^{\prime}, V\right)$ as $f=\sum f_{k} s_{k}$, and write its Bergman projection as $P f=$ $\sum(P f)_{k} s_{k}$. Then there is a compact operator $K: L^{2}\left(W^{\prime}, V\right) \rightarrow L^{2}(W, V)$ so that, for each $z \in W$,

$$
(P f)_{k}(z)=\int_{W^{\prime}} \frac{\lambda(z) f_{k}(w)}{(1-R(z, w))^{n+1}} d V(w)+(K f)_{k}(z) .
$$

After multiplying (18) by $s_{k}$ and summing, we obtain the same formula without the subscript $k$. Note also that the singularity of the integral kernel in (18) occurs only on the boundary diagonal. This follows from the (SGCS) inequality.

\section{The integral operator}

We return to the situation where $B$ denotes the unit disk bundle. By the negativity of $L$, it is a strongly pseudoconvex domain, defined by the inequality $R(z, z)<1$. After using a partition of unity we will be able to apply Proposition 4 .

According to Proposition 1 we want to show that $G R^{d}$ is the kernel of a positive operator for sufficiently large $d$. To do so, we consider the operator whose kernel is $\sum_{0}^{\infty} m_{d} G R^{d}$, where the $m_{d}$ are positive numbers. By choosing them equal to the appropriate binomial coefficients, we have

$$
\sum_{0}^{\infty} m_{d} G(z, w) R(z, w)^{d}=c \frac{G(z, w)}{(1-R(z, w))^{n+1}},
$$

and this begins to look like the Bergman kernel for the domain $R(z, z)<1$. Motivated by this discussion we define the crucial integral operator $\mathcal{T}$ on $L^{2}\left(B, \mathcal{E}^{*}\right)$ by

$$
\mathcal{T}(f)(z)=\int_{B} \frac{\langle f(w), \overline{\mathcal{G}(z, w)}\rangle}{(1-R(z, w))^{n+1}} d V(w)
$$

The next lemma states the basic estimate we need to prove about $\mathcal{T}$.

Lemma 1. Let $T$ be a bounded linear operator on $L^{2}$, and suppose that $A^{2}$ is a countable orthogonal sum of subspaces $V_{j}$. Suppose that there is a positive constant $c$ and a compact operator $K$ on $L^{2}$ such that, for all $h \in A^{2}$,

$$
\langle T h, h\rangle \geq c\|h\|^{2}-\langle K h, h\rangle .
$$


Then there is an integer $d$ so that, for all $j \geq d$, the restriction of $T$ to $V_{j}$ is positive. For $h \in V_{j} \subset A^{2}$, we have $\langle T h, h\rangle \geq \frac{c}{3}\|h\|^{2}$.

Proof. Our hypothesis states that $T+K=Q$ where $Q$ is positive. We write $\|\mid \mathcal{L}\|$ for the operator norm of $\mathcal{L}$. Since $K$ is compact, there is an operator $\mathcal{L}$ with finite-dimensional range such that $\left\||K-\mathcal{L} \||<\frac{c}{3}\right.$. We write $T=Q-K=$ $Q-(K-\mathcal{L})-\mathcal{L}$. Since $\mathcal{L}$ is finite rank, for sufficiently large $j$ we may assume that the restriction of $\mathcal{L}$ to $V_{j}$ has operator norm at most $\frac{c}{3}$. We obtain, for such $j$ and $h \in V_{j}$,

$\langle T h, h\rangle \geq c\|h\|^{2}-\langle(K-\mathcal{L}) h, h\rangle-\langle\mathcal{L} h, h\rangle \geq c\|h\|^{2}-\frac{c}{3}\|h\|^{2}-\frac{c}{3}\|h\|^{2}=\frac{c}{3}\|h\|^{2}$,

By (21) $T$ is positive on $V_{j}$.

\section{Proof of Theorem 1}

First we prove inequality (20) for the operator $\mathcal{T}$. This takes most of the effort. Then we close this section by completing the proof of Theorem 1 .

To prove inequality (20) for the operator $\mathcal{T}$, we require a lemma describing what sorts of error terms define compact operators.

Lemma 2. Suppose that $\Omega=\{R(z, z)<1\}$ is strongly pseudoconvex, and that $R$ satisfies (SGCS). Suppose that $q$ is a smooth function on $\bar{\Omega} \times \bar{\Omega}$ that vanishes on the diagonal. Then the operator $Q$ defined by

$$
Q f(z)=\int_{\Omega} \frac{q(z, w)}{(1-R(z, w))^{n+1}} f(w) d V(w)
$$

is compact on $L^{2}$.

Proof. The only possible singularities of the kernel occur when $z=w$ on the boundary, because the (SGCS) inequality holds. Since the numerator vanishes there, this compensates for them. There are two ways to prove this. In both proofs we may assume that we are working in $\mathbb{C}^{n}$ after using a partition of unity.

In the first proof we compare the factor $\frac{1}{(1-R(z, w))^{n+1}}$ with the singularity of the Bergman kernel. We follow the proof of Theorem 1 in [CD2]; the proof is easier because the analogue of Lemma 3 from that paper is well known for strongly pseudoconvex domains.

The second proof shows directly from the generalized Young's inequality that the operator $Q$ is the limit in the operator norm of compact operators, and hence is itself compact. We sketch this proof here.

In order to show that $Q$ is compact, we will show that, given $\epsilon>0$, there is a compact operator $Q_{\epsilon}$ so that $\left\|Q Q-Q_{\epsilon}\right\| \| \rightarrow 0$ as $\epsilon \rightarrow 0$. Given $\epsilon$, we let $\phi$ be a smooth positive function, bounded above by unity, that is identically one near 
the origin and is supported in a ball of radius $\epsilon$. Let us write the integral kernel in (22) as $A(z, w)$ Then

$$
A(z, w)=\phi(w-z) A(z, w)+(1-\phi(w-z)) A(z, w) .
$$

The second term in (23) is the kernel of a compact operator, because it is smooth everywhere. We will finish the proof by showing that the first term, called $I_{\epsilon}(z, w)$, defines a kernel whose operator norm is less than a constant times $\epsilon^{\frac{1}{2}}$.

The estimate for the operator norm of $I_{\epsilon}(z, w)$ uses the generalized Young's inequality [Fo]: If we show that there is a constant $C$ so that, for all $z \in \Omega$,

$$
\int_{\Omega}\left|I_{\epsilon}(z, w)\right| d V(w) \leq C \epsilon^{\frac{1}{2}}
$$

and we also show the analogous inequality with $z$ and $w$ interchanged, then $I_{\epsilon}(z, w)$ is the kernel of a bounded operator on $L^{2}$ with operator norm $\left\|\left|I_{\epsilon}\right|\right\| \leq$ $C \epsilon^{\frac{1}{2}}$.

The two estimates are similar; we prove (24). Fix $z \in \Omega$. Let $\Omega_{\epsilon}=\{|w-z|<$ $\epsilon\} \cap \Omega$. By the condition on the support of $\phi$ we have

$$
\int_{\Omega}\left|I_{\epsilon}\right| d V(w)=\int_{\Omega_{\epsilon}}|\phi(w-z) A(z, w)| d V(w) .
$$

Therefore

$$
\int_{\Omega}\left|I_{\epsilon}\right| d V(w) \leq \int_{\Omega_{\epsilon}}|A(z, w)| d V(w)=\int_{\Omega_{\epsilon}}|q(z, w)| \mid\left(1-\left.R(z, w)\right|^{-n-1} d V(w) .\right.
$$

We estimate $|q(z, w)|$ for $|w-z|<\epsilon$ using $|q(z, w)| \leq m|w-z| \leq m \epsilon^{\frac{1}{2}}|w-z|^{\frac{1}{2}}$.

We see that the left side of $(26)$ can be estimated by $m \epsilon^{\frac{1}{2}}$ times an explicit integral. Writing $w=z+t \zeta$ where $\zeta$ is on the unit sphere, and integrating over $\{|t \zeta|<\epsilon\} \cap \Omega$ gives a finite value independent of $z$.

An immediate consequence of Lemma 2 is that kernels of the form

$$
\frac{q(z)-q(w)}{(1-R(z, w))^{n+1}}
$$

(where $q$ is smooth) define compact operators.

In order to use formula (18), we first let $W_{\nu}$ denote an open covering of $B$ over which the bundle $\mathcal{E}^{*}$ is trivial. Next let $\chi_{\nu}$ denote smooth non-negative functions compactly supported in $W_{\nu}$ such that $\sum_{\nu}\left(\chi_{\nu}\right)^{2}=1$. (The square is 
convenient because Lemma 1 considers a quadratic form.) We let $W_{\nu}^{\prime} \subset \subset W_{\nu}$ be relatively compact domains containing the supports of the $\chi_{\nu}$. We have

$$
\langle\mathcal{T} f, f\rangle=\sum_{\nu}\left\langle\left(\chi_{\nu}\right)^{2} \mathcal{T} f, f\right\rangle=\sum_{\nu}\left\langle\chi_{\nu} \mathcal{T} f, \chi_{\nu} f\right\rangle
$$

In (27), we write $\chi_{\nu}(z)=\left(\chi_{\nu}(z)-\chi_{\nu}(w)\right)+\chi_{\nu}(w)$. Each term is multiplied by a smooth function divided by $(1-R(z, w))^{n+1}$. By Lemma 2 the first such term defines a compact operator; it corresponds to the commutator $\left[\chi_{\nu}, \mathcal{T}\right]$. The second term corresponds to the operator $\mathcal{T} \chi_{\nu}$. Thus, with $K$ compact, we may write

$$
\langle\mathcal{T} f, f\rangle=\sum\left\langle\mathcal{T} \chi_{\nu} f, \chi_{v} f\right\rangle+\langle K f, f\rangle
$$

It therefore suffices to prove the basic estimate corresponding to the quadratic form given by $\sum_{\nu}\left\langle\mathcal{T} \chi_{\nu} f, \chi_{v} f\right\rangle$. According to the definition of $\mathcal{T}$, we have

$$
\left\langle\mathcal{T} \chi_{\nu} f, \chi_{\nu} f\right\rangle=\int_{B} \int_{B} \frac{\left\langle\left\langle\chi_{\nu}(w) f(w), \overline{\mathcal{G}(z, w)}\right\rangle, \chi_{\nu}(z) f(z)\right\rangle}{(1-R(z, w))^{n+1}} d V(w) d V(z) .
$$

In (29) we can use $W_{\nu}$ for the region of integration and express the integrand in terms of the local frame. We write $f=\sum f_{k} s_{k}$ and $\mathcal{G}(z, w)=$ $\sum_{i j} \mathcal{G}_{j i}(z, w) s_{i}(z) \otimes \overline{s_{j}(w)}$. These coefficients depend on the index $\nu$. This gives

$$
\begin{aligned}
& \int_{W_{\nu}} \int_{W_{\nu}} \chi_{\nu}(w) \chi_{\nu}(z) \\
& \quad \sum_{i, j, k, l} \frac{f_{k}(w) \overline{f_{l}(z)} \mathcal{G}_{j i}(z, w)\left\langle\left\langle s_{k}(w), \overline{s_{i}(z)} \otimes s_{j}(w)\right\rangle, s_{l}(z)\right\rangle}{(1-R(z, w))^{n+1}} d V(w) d V(z) .
\end{aligned}
$$

In (30) we write $D_{k j}$ for $\left\langle s_{k}, s_{j}\right\rangle$ (this also depends on $\nu$ ) to obtain

$$
\begin{aligned}
& \left\langle\mathcal{T} \chi_{\nu} f, \chi_{\nu} f\right\rangle= \\
& \int_{W_{\nu}} \int_{W_{\nu}} \chi_{\nu}(w) \chi_{\nu}(z) \sum_{i, j, k, l} \frac{f_{k}(w) \overline{f_{l}(z)} \mathcal{G}_{j i}(z, w) D_{k j}(w) \overline{D_{l i}(z)}}{(1-R(z, w))^{n+1}} d V(w) d V(z) .
\end{aligned}
$$

In (31) we write $D_{j k}(w)=\left(D_{j k}(w)-D_{j k}(z)\right)+D_{j k}(z)$ and apply Lemma 2 as before. The first terms introduce a compact operator, so we consider

$$
\int_{W_{\nu}} \int_{W_{\nu}} \chi_{\nu}(w) \chi_{\nu}(z) \sum \frac{f_{k}(w) \overline{f_{l}(z)} \mathcal{G}_{j i}(z, w) D_{k j}(z) \overline{D_{l i}(z)}}{(1-R(z, w))^{n+1}} d V(w) d V(z)
$$


We perform the same trick on $(32)$, writing $\mathcal{G}_{j i}(z, w)=\left(\mathcal{G}_{j i}(z, w)-\mathcal{G}_{j i}(z, z)\right)+$ $\mathcal{G}_{j i}(z, z)$. Again the first term introduces a compact error, so we now consider

$$
\int_{W_{\nu}} \int_{W_{\nu}} \frac{\chi_{\nu}(w) \chi_{\nu}(z) \sum f_{k}(w) \overline{f_{l}(z)} \mathcal{G}_{j i}(z, z) D_{k j}(z) \overline{D_{l i}(z)}}{(1-R(z, w))^{n+1}} d V(w) d V(z)
$$

We multiply and divide by the positive factor $\lambda(z)$. We write

$$
A_{k l}^{\nu}(z)=\frac{\sum_{j, i} \mathcal{G}_{j i}(z, z) D_{k j}(z) \overline{D_{l i}(z)}}{\lambda(z)},
$$

to simplify (33).

$$
\int_{W_{\nu}} \int_{W_{\nu}} \frac{\chi_{\nu}(w) \chi_{\nu}(z) \sum_{k, l} A_{k l}^{\nu}(z) \lambda(z) f_{k}(w) \overline{f_{l}(z)}}{(1-R(z, w))^{n+1}} d V(w) d V(z) .
$$

The inner integral, modulo compact errors, equals $\left(P \chi_{\nu} f\right)_{k}(z)$ by Proposition 4 .

Because $\mathcal{G}_{j i}(z, z)$ defines a metric, and because $\lambda(z)$ is positive, the matrix $A_{k l}^{\nu}(z)$ is positive definite. Combining these gives, for some compact operator $K_{1}$,

$$
\left\langle\mathcal{T} \chi_{\nu} f, \chi_{\nu} f\right\rangle=\int_{W_{\nu}} \sum_{k, l} A_{k l}^{\nu}(z) \chi_{\nu}(z)\left(P \chi_{\nu} f\right)_{k}(z) \overline{f_{l}(z)} d V(z)+\left\langle K_{1} f, f\right\rangle .
$$

Suppose that $f \in A^{2}\left(B, \mathcal{E}^{*}\right)$. Then we may substitute $\chi_{\nu}(z) f_{k}(z)$ for $P\left(\chi_{\nu} f\right)_{k}(z)$ in $(35)$ again making a compact error. This gives, for some compact operator $K$

$$
\left\langle\mathcal{T} \chi_{\nu} f, \chi_{\nu} f\right\rangle=\int_{W_{\nu}} \sum_{k, l} A_{k l}^{\nu}(z)\left(\chi_{\nu}(z)\right)^{2} f_{k}(z) \overline{f_{l}(z)} d V(z)+\langle K f, f\rangle
$$

Since $A_{k l}^{\nu}$ is positive definite we bound the sum below using

$$
\sum_{k, l} A_{k l}^{\nu}(z) f_{k}(z) \overline{f_{l}(z)} \geq c_{\nu}\langle f(z), f(z)\rangle
$$

Summing (37) on $\nu$ (we assume that this is a finite sum), we obtain inequality (20) for the operator $\sum_{\nu} \chi_{\nu} \mathcal{T} \chi_{\nu}$. By (28) we obtain inequality (20) also for $\mathcal{T}$.

We can now finish the proof of Theorem 1 . We now write $A^{2}$ as the orthogonal sum of the spaces $\mathcal{H}^{j}\left(B, \mathcal{E}^{*}\right)$. They are orthogonal because we have chosen the metric on $\mathcal{E}^{*}$ to be invariant under the circle action. We have shown that, for 
sufficiently large $j$, the restriction of $\mathcal{T}$ to $\mathcal{H}^{j}\left(B, \mathcal{E}^{*}\right)$ is positive. On the other hand for appropriate positive constants we can write

$$
\frac{1}{(1-R(z, w))^{n+1}}=\sum_{j=0}^{\infty} m_{j} R(z, w)^{j},
$$

and we see that the restriction of $\mathcal{T}$ to $\mathcal{H}^{j}\left(B, \mathcal{E}^{*}\right)$ is a positive constant times the integral operator whose kernel is $G(z, w) R(z, w)^{j}$. Expressing this kernel in terms of a basis of sections of $\mathcal{H}^{j}\left(B, \mathcal{E}^{*}\right)$ shows that the underlying matrix of coefficients is positive-definite. By Propositions 1 and 3 , we obtain the conclusion of Theorem 1.

\section{Another metric on $E \otimes L^{d}$.}

In [CD2] the authors proved the following result:

Theorem [CD2]. Suppose that $\Omega$ is a smoothly bounded pseudoconvex circled domain in $\mathbb{C}^{n}$ of finite type. For each integer $d$, let $\Phi^{d}=\left(\Phi_{1}^{d}, \ldots, \Phi_{N}^{d}\right)$ denote an orthonormal basis for the homogeneous polynomials of degree $d$ on $\Omega$. Let $f$ be a bihomogeneous polynomial that is positive away from the origin. Then there is an integer $d_{0}$ (depending on $f$ ) such that, for each $d \geq d_{0}$, there is a homogeneous polynomial mapping $h$ such that

$$
\left\|\Phi^{d}(z)\right\|^{2} f(z, \bar{z})=\|h(z)\|^{2} .
$$

This result can be rephrased in the language of the current paper. As before $f$ defines a metric on some $\mathbb{U}^{m}$ over projective space. The function $\left\|\Phi^{d}(z)\right\|^{2}$ defines a metric on $\mathbb{U}^{d}$, and their product defines a metric on $\mathbb{U}^{m+d}$. When $\Omega$ is the unit ball, $\left\|\Phi^{d}(z)\right\|^{2}$ differs only in certain constants from $\|z\|^{2 d}$. In general Theorem [CD2] differs in that $\Omega$ is allowed to be weakly pseudoconvex, and that the metric on $\mathbb{U}^{m+d}$ is not the natural one considered in Theorem 1 of this paper.

It is possible to give a version of Theorem 1 that generalizes Theorem [CD2]. We state the result but omit the proof. Note that it is not necessary here to assume that the (SGCS) holds. The proof is similar, as the hypotheses enable one to involve the Bergman kernel more easily. On the other hand one needs other information about the Bergman projection that follows from subelliptic estimates for the $\bar{\partial}$-Neumann problem.

Theorem 2. Suppose that $M$ is a compact complex manifold, that $E$ is a vector bundle over $M$ with a globalizable metric $G$, and that $L$ is a line bundle over $M$ with globalizable metric $R$. Let $\left\|\phi_{d}\right\|^{2}$ denote the metric on $L^{d}$ arising from an orthonormal basis for $A^{2}$ sections of $\mathcal{E}^{*}$ over the unit disk bundle B. Suppose that $B$ is pseudoconvex and finite type. Then there is an integer $d$ and a holomorphic mapping $h_{d}$ so that $E \otimes L^{d}=h^{*}\left(\mathbb{U}_{p, N}\right)$ and $\left\|\phi_{d}\right\|^{2} G=h^{*}\left(g_{0}\right)$. 


\section{Positivity versus Non-negativity}

Artin's solution of Hilbert's problem shows that a homogeneous polynomial on real Euclidean space that is non-negative can be written as a sum of squares of rational functions. It is false that a bihomogeneous polynomial on complex Euclidean space that assumes non-negative values must be a quotient of squared norms. The simplest counterexample is given by equation (38), but a more interesting one is given in (39).

$$
\begin{aligned}
& p(z, \bar{z})=\left(\left|z_{1}\right|^{2}-\left|z_{2}\right|^{2}\right)^{2} . \\
& h(z, \bar{z})=\left(\left|z_{1} z_{2}\right|^{2}-\left|z_{3}\right|^{4}\right)^{2}+\left|z_{1}\right|^{8} .
\end{aligned}
$$

The polynomial $p$ from (38) is not a quotient of squared norms because its zero set fails to be a complex variety. The zero set of the polynomial $h$ is the complex variety defined by setting $z_{1}=z_{3}=0$, but $h$ is not a quotient of squared norms. The reason is that it doesn't satisfy a more intriguing necessary condition, called the jet pullback property. Suppose that $h(z, \bar{z})=\|A(z)\|^{2} /\|B(z)\|^{2}$ for holomorphic polynomial mappings $A$ and $B$. Let $t$ be a complex variable, and let $t \rightarrow z(t)$ be a holomorphic mapping. It is easy to see that the lowest order term in the Taylor expansion of $t \rightarrow h(z(t))$ must then be independent of the argument of $t$. When $h$ is given by (39), however, this property fails. Consider for example the mapping given by $z(t)=\left(t^{2}, 1+t, t\right)$. Then the pullback $h(z(t), \overline{z(t)})$ vanishes to order ten at the zero, but the terms of order ten include $t^{4} \bar{t}^{6}$.

These examples show that the analogue of Hilbert's seventeenth problem for complex variables requires more than non-negativity. Our result from [CD1] solves the problem for polynomials that are positive away from the origin, but we have not yet succeeded in establishing necessary and sufficient conditions for the non-negativity of a bihomogeneous polynomial.

\section{Concluding Remarks}

Factorization of operator-valued analytic functions of one complex variable was studied extensively in the early 1970s. See [RR] for example. The idea of multiplying the operator by a high power of a scalar operator before attempting a factorization seems not to have arisen then. Another classical result on factorization of Hermitian matrices of polynomials appears in [Dj]. Although that result considers polynomials in several variables, it gives a positive result only in very special cases. That paper does not consider the possibility of multiplying the matrix by high powers of a scalar either.

On the other hand, high tensor powers of line bundles often arise in algebraic geometry and complex manifold theory, especially in regard to imbeddings into 
Grassman manifolds. In this sense the result in this paper can be considered an isometric relative of the Kodaira imbedding theorem. In 1953 Calabi [CL] studied isometric imbeddings for Kähler manifolds. Thus the bundle involved is the tangent bundle. Calabi uses the Kähler potential to construct a real analytic-function $D: M \times M \rightarrow \mathbb{C}$ called the diastatic function. This function is holomorphic in the first variable, Hermitian in the sense of Definition 1, and therefore anti-holomorphic in the second variable. One of the conditions for imbedding (into Fubini-Study spaces) is the singled-valued nature of this function in one variable for all choices of the other. The condition that $D(z, w)$ vanishes only when $z=w$ arises in guaranteeing the topological nature of the imbedding. This condition is analogous to our (SGCS).

\section{References}

[BS] L. Boutet de Monvel and J. Sjöstrand, Sur la singularite des noyaux de Bergman et Szegö, Journées: Équations aux Dérivées Partielles de Rennes (1975), Asterisque, no. 34-35, Soc. Mat. de France, Paris, 1976, pp. 123-164.

[CL] E. Calabi, Isometric imbedding of complex manifolds, Ann. of Math. (2) 58 (1953), $1-23$.

[C] D.W. Catlin, The Bergman kernel and a theorem of Tian, Analysis and Geometry in Several Complex Variables, Proceedings of the Taniguchi Symposium in Complex Analysis (M. Kuranishi and G. Komatsu, ed.), Birkhauser, to appear.

[CD1] D.W. Catlin and J.P. D'Angelo, A stabilization theorem for Hermitian forms and applications to holomorphic mappings, Math. Res. Lett. 3 (1996), 149-166.

[CD2] , Positivity conditions for bihomogeneous polynomials, Math. Res. Lett. 4 (1997), 1-13.

[De] J.-P. Demailly, $L^{2}$ vanishing theorems for positive line bundles and adjunction theory, CIME Session, Transcendental Methods in Algebraic Geometry, Cetraro, Italy, 1994.

[Dj] D.Z. Djokovic, Hermitian matrices over polynomial rings, J. Algebra 43 (1976), 359374 .

[Fe] C. Fefferman, The Bergman kernel and biholomorphic mappings of strongly pseudoconvex domains 26 (1974), Invent. Math., 1-65.

[Fo] G.B. Folland, Introduction to partial differential equations, Princeton University Press, Princeton, NJ, 1976.

[Q] D.G. Quillen, On the Representation of hermitian forms as sums of squares, Invent. Math. 5 (1968), 237-242.

[RR] M. Rosenblum and J. Rovnyak, The factorization problem for nonnegative operator valued functions, Bull. Amer. Math. Soc. 77 (1971), 287-318.

[R] B. Reznick, Some concrete aspects of Hilbert's 17th Problem, to appear in the RAGOS Proceedings, Contemporary Math. (1998), also available on the World Wide Web from http: //www.math.uiuc.edu/reznick/.

[W] R.O. Wells, Differential analysis on complex manifolds, Prentice-Hall, Englewood Cliffs, New Jersey, 1973.

Deptartment of Mathematics, Purdue University, W. Lafayette, IN 47907

E-mail address: catlin@math.purdue.edu

Dept. of Mathematics, Univ. of Illinois, Urbana IL 61801

E-mail address: jpda@math.uiuc.edu 